\title{
A Cross-sectional Analysis of Glove Perforation in Primary and Revision Total Hip Arthroplasty
}

\author{
Kumar D, MS, Shantanu K, MCh, Kumar M, MS, Kumar A, MCh, Sharma V, FICS \\ Department of Orthopaedics, King George's Medical University, Lucknow, India
}

\author{
(c) (1) \\ Date of submission: July 2016 \\ Date of acceptance: October 2016
}

\begin{abstract}
The number of total hip arthroplasties is ever increasing. Literature about glove perforation rates in arthroplasties in India is very scarce. The purpose of our study was to determine the incidence of glove perforation and increasing the awareness of possible glove perforations to decrease the risk of infection. We performed a prospective study in which we tested gloves worn by all scrubbed personnel. A total of 1408 gloves were collected from 42 primary total hip and 13 revision total hip arthroplasties. Incidence of glove perforation was found to be more in revision total hip arthroplasty. We found a greater outer glove perforation rate of about $38.33 \%$ as compared to $25 \%$ inner glove perforation rate. Outer glove perforation was recognized $100 \%$ of time intraoperatively but inner glove perforation was noted only $17 \%$ of time. First assistant recorded highest rate of glove perforation.
\end{abstract}

\section{Key Words:}

Surgical gloves, perforation, primary total hip arthroplasty, revision total arthroplasty, latex

\section{INTRODUCTION}

Total hip arthroplasty is a very rewarding surgery. As the number of total hip arthroplasties is increasing day by day ${ }^{1}$, revision total hip surgeries are also increasing. In the Western literature infection rates are found to be $0.88 \%$ to $0.92 \%$ for primary arthroplasty and $2.9 \%$ to $30.2 \%$ for revision arthroplasty ${ }^{1-7}$. Periprosthetic infections are among the most devastating complications for the patients as well as for the surgeons. The etiology of periprosthetic infections is multifactorial. Diabetes and poor socioeconomic status are the major patient related factors, while major surgical factors include operative time and allogenic blood transfusion whereas postoperative complications such as urinary tract infection, myocardial infarction and longer duration of hospitalization have also been attributed to increased risk of periprosthetic infections ${ }^{8}$. Laminar air flow and prophylactic antibiotics are commonly used by the surgeons to decrease the risk of perioperative infections ${ }^{8-10}$. Despite all the efforts made by surgeons strict aseptic technique is essential to minimize the risk of surgical field contamination.

The reported incidence of glove perforation in orthopaedic procedures is found to be between $3.6 \%$ and $26 \%{ }^{11-18}$. Previous studies have shown an association between glove perforation and duration of procedure, hand dominance, and specific portions of procedure $11,12,1,19$. The present authors have not found any studies in India that specifically evaluate incidence of glove perforation in primary and revision total hip arthroplasty.

This cross-sectional, comparative study was designed to evaluate the incidence of glove perforation in primary and revision total hip arthroplasty. The main objective was to compare the incidence of glove perforation in primary and revision total hip arthroplasty. The secondary objective was to study the factors responsible for glove perforation in total hip arthroplasty and to increase the awareness of surgeons to glove perforation in total hip arthroplasty. We hypothesized that the rate of glove perforation is greater in revision total hip as compared to primary total hip arthroplasty.

\section{MATERIALS AND METHODS}

This study was conducted during the period from June 2014 to May 2015 at our institute. Natural rubber latex, textured and non-powdered gloves (B.Braun Medical Ltd.) which is routinely provided by the University, were used in the study. A single arthroplasty team of surgeons performed all the surgeries to maintain the same techniques and standards during surgery. We followed triple-gloving protocol for all of surgeries in the study. The outermost (preparation) layer used for draping purposes and then discarded. The second layer was worn throughout the procedure and changed as and when required. The first innermost layer was worn right through unless a perforation was noted.

Usually a team of five or six personnel were scrubbed in a 
Table I: Rates of glove perforation according to scrubbed personnels

\begin{tabular}{|c|c|c|c|c|c|}
\hline Particular & Perforation & $\%$ & Non Perforated & $\%$ & Total used \\
\hline \multicolumn{6}{|c|}{ Chief Surgeon } \\
\hline Prep gloves & 1 & 2.38 & 41 & 97.62 & 42 \\
\hline Outer gloves & 15 & 10.48 & 128 & 89.52 & 143 \\
\hline Inner gloves & 2 & 1.78 & 110 & 98.22 & 112 \\
\hline \multicolumn{6}{|c|}{ First Assistant } \\
\hline Prep gloves & 3 & 2.65 & 110 & 97.35 & 113 \\
\hline Outer gloves & 29 & 16.38 & 148 & 83.62 & 177 \\
\hline Inner gloves & 13 & 10.57 & 110 & 89.43 & 123 \\
\hline \multicolumn{6}{|c|}{ Second Assistant } \\
\hline Prep gloves & 2 & 1.79 & 110 & 98.21 & 112 \\
\hline Outer gloves & 10 & 7.81 & 118 & 92.19 & 128 \\
\hline Inner gloves & 5 & 4.35 & 110 & 95.65 & 115 \\
\hline \multicolumn{6}{|c|}{ Scrub Nurse } \\
\hline Prep gloves & 2 & 1.79 & 110 & 98.21 & 112 \\
\hline Outer gloves & 6 & 5.41 & 105 & 94.59 & 111 \\
\hline Inner gloves & 0 & 0 & 110 & 100 & 110 \\
\hline
\end{tabular}

Table II: Outer gloves perforations: Primary vs Revision THA

\begin{tabular}{|c|c|c|c|c|c|c|}
\hline Particular & Perforation & $\%$ & Non perforated & $\%$ & Total used & $p$ value \\
\hline \multicolumn{7}{|c|}{ Chief Surgeon } \\
\hline Primary THA & 13 & 5.49 & 224 & 94.51 & 237 & 0.689 \\
\hline Revision THA & 5 & 7.81 & 59 & 92.19 & 64 & \\
\hline \multicolumn{7}{|c|}{ First Assistant } \\
\hline Primary THA & 28 & 8.67 & 295 & 91.13 & 323 & 0.01 \\
\hline Revision THA & 17 & 18.89 & $\begin{array}{c}73 \\
\text { Second Assistant }\end{array}$ & 81.11 & 90 & \\
\hline Primary THA & 10 & 3.65 & 264 & 96.35 & 274 & 0.148 \\
\hline Revision THA & 7 & 8.24 & 78 & 91.76 & 85 & \\
\hline \multicolumn{7}{|c|}{ Scrub Nurse } \\
\hline Primary THA & 6 & 2.37 & 247 & 97.63 & 253 & 0.972 \\
\hline Revision THA & 2 & 2.44 & 80 & 97.56 & 82 & \\
\hline
\end{tabular}

Table III: Glove perforations noticed according to stage of operation

\begin{tabular}{|lcc|}
\hline & Number & $\%$ \\
\hline Setting up & 2 & 6.06 \\
Preparation of bone & 25 & 75.75 \\
Closure & 6 & 18.19 \\
Total & 33 & \\
\hline
\end{tabular}

Table IV: Total number of gloves perforations and awareness of perforations

\begin{tabular}{|lccc|}
\hline Combined & Perforations & Noticed & $\%$ \\
\hline Prep gloves & 8 & 5 & 62.5 \\
Outer gloves & 60 & 23 & 38.33 \\
Inner gloves & 20 & 5 & 25.00 \\
Total & 88 & 33 & 37.50 \\
\hline
\end{tabular}

procedure. We collected all the data regarding scrubbed personnel before each procedure such as role of the person during the case and hand dominance, after having obtained an informed consent from all. At the end of each procedure we collected all the gloves, labeled individually and placed in marked plastic bags with details of the person who had worn those gloves. At time of gloves removal, data such as glove material, layer, side, duration of wear, time of removal and reason of glove removal were recorded.

At completion of each procedure all the collected gloves were reexamined for perforations using standardized water infusion method described by the American Society for Testing and Material Guidelines ${ }^{20}$. We tested the gloves by 


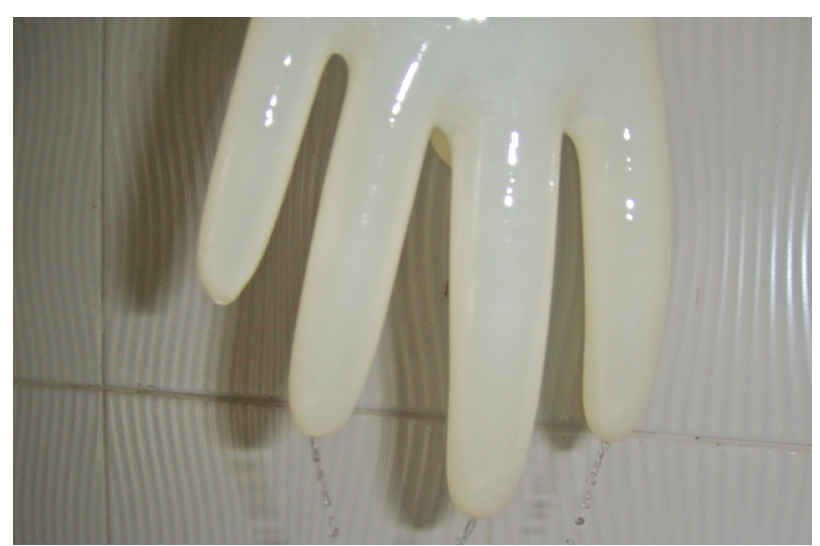

Fig. 1: Identification of perforations by pressurization of the gloves filled with water.

filling them with $1000 \mathrm{ml}$ of water and suspended from the occluded cuff, $5 \mathrm{ft}$ from the ground (Fig. 1) .The gloves and the digits were pressurized and all the perforations were identified by a jet of water ${ }^{20}$. Perforation were noted as per their location, size, number and cause.

Statistical analysis of data was conducted to evaluate significant association with the factors analyzed and perforations using the Fisher exact test. $\mathrm{P}$ value less than .05 were considered statistically significant.

\section{RESULT}

All the gloves were collected from 42 primary and 13 revision total hip arthroplasties performed at our institute during the period of study. A total one thousand four hundred and eight gloves were retrieved from all the scrubbed up personnel. The total incidence of glove perforation was found to be $6.25 \%(88 / 1408)$ if all gloves were included. We examined the gloves of the chief surgeon, first assistant, second assistant and scrub nurse. The number of perforations related to glove layer and seniority of scrubbed personnel are listed in Table I. Outer glove perforations were noticed in $38.33 \%(23 / 60)$ whereas inner glove perforations were noticed only $25 \%(5 / 20)$. Total glove perforations of the chief surgeon was noted to be $6.06 \%(18 / 297)$. Out of all scrubbed personnel maximum glove perforations encountered were with the first assistant $10.9 \%(45 / 413)$ and that was inclusive of all three layers. Probably the reason behind this was that the assistant was involved in performing the parts of the procedure most at risk to glove perforation. The first assistant had a 14\% (42/300) combined outer and inner glove perforation rate in all cases. Outer gloves perforated in $16.38 \%(29 / 177)$ and inner gloves in $10.57 \%(13 / 123)$.

Comparison of incidence of glove perforation in primary and revision THA is presented in Table II. Total glove perforations occurred in 5.24\% (57/1087) worn during primary THA compared with $9.65 \%(31 / 321)$ in revision
THA. The inner layer was perforated in 4.34\% (20/460) of all THA cases. Outer gloves had an incidence of $10.73 \%$ $(60 / 559)$ of all THA cases. The first assistant had outer glove perforation in $8.67 \%(28 / 323)$ of primary THA compared with $18.89 \%(17 / 90)$ in revision cases $(\mathrm{P}=.01)$.

In primary and revision THA cases, total $75.75 \%(25 / 33)$ of perforations occurred from exposure to preparation of bone (Table III). Total of 88 perforations were found in all the collected gloves out of which only $37.5 \%$ (33/88) had been noticed during the procedure and gloves changed accordingly. Most of the perforations noticed in the preparation layer and outer layer of gloves $31.81 \%$ (28/88). Inner layer perforations were occasionally noticed (Table IV).

Analysing the time duration of wearing of the gloves there was no significant difference between perforated and nonperforated gloves in the group of scrubbed personnel. The mean time recorded was $(53+-17 \mathrm{~min})$. Sixty three percent perforations were found on the index finger, followed by $20 \%$ on the thumb, while most of the $(76 \%)$ perforations occurred on the non-dominant hand.

\section{DISCUSSIONS}

During any surgical procedure gloves act as a vital component in maintaining the barrier between surgical team and the patient. Gloves reduce the chances of risk of disease transmission and subsequent infection ${ }^{21-24}$. For this reason glove perforation is a serious complication that needs to be addressed vigorously because it exposes both the surgical team as well as the patient to the risk of infection. This study is the first of its type in the Indian setup to demonstrate the incidence of glove perforation in primary as well as revision THA. Furthermore, this study is important to enhance the awareness of glove perforation as sufficient number of perforations were not noticed by the surgical team in most of the cases.

We used a double gloved protocol in all the cases while a third (outer) layer of gloves were used for draping and preparation (preparation layer). Overall 6.25\% gloves were found to be perforated among all the scrubbed personnel. Perforations were recorded in outer gloves in $10.73 \%$ and $4.34 \%$ perforations were found in the inner gloves. The reported incidence of glove perforations in the Western literature ranges from $6.8 \%$ to $14.6 \%$ in THA and TKA ${ }^{17,25}$.

We found a significant increase in the incidence of glove perforations of the first assistant during revision THA as compared to primary THA and this was mainly because of the more complex procedures with increased duration of surgery and greater exposure to sharp bone and metal. 
This study was conducted with many limitations. The foremost limitation was of unnoticed perforations; however, the scrubbed persons had changed the gloves time to time but it was still not possible to detect every unnoticed perforation. The second limitation was of the water infusion method that was used to detect perforations. This method used to overdistend the gloves, causing water to come out through the perforation in jet form. In this method the main concern was that pressurization might have caused perforations in the gloves where structural integrity was probably compromised but actual perforation had not existed before testing.

There was no significant difference between the wearing time duration of perforated and non-perforated gloves which was probably because of routine changing of gloves at different stages of the procedure. However two studies reported previously that the incidence of glove perforations increases with duration of glove wear ${ }^{26}$. Most perforations occurred on the index finger of non-dominant hand, these findings are supported by previous studies as well.

\section{CONCLUSION}

Increasing the awareness for glove perforations is very vital in preventing disease transmission or contamination between patient and operating room personnel. Most of the glove perforations go unnoticed, so a practical guideline for wearing the gloves, duration of glove wearing and assessment of glove perforation should be formulated. Orthopaedic procedures especially arthroplasties bear a higher risk of glove perforations, more so with revision surgeries. Therefore when a perforation is detected removal and a careful inspection of inner layer before regloving with a new outer layer is required.

\section{REFERENCES}

1. Kurtz S, Mowat F, Ong K, Chan N, Lau E, Halpern M. Prevalence of primary and revision total hip and knee arthroplasty in the United States from 1990 through 2002. J Bone Joint Surg Am. 2005; 87: 1487.

2. Kurtz SM, Lau E, Schmier J, Ong KL, Zhao K, Parvizi J. Infection burden for hip and knee arthroplasty in the United States. $J$ Arthroplasty. 2008; 23: 984.

3. Philips C, Barrett JA, Losina E, Mahomed NN, Lingard EA, Guadagnoli E, et al. Incidence rates of dislocation, pulmonary embolism, and deep infection during the first six months after elective total hip replacement. J Bone Joint Surg. 2003; 85: 20.

4. Springer BD, Fehring TK, Griffin WL, Odum SM, Masonis JL. Why revision total hip arthroplasty fails. Clin Orthop Relat Res. 2009; 467: 166.

5. Jafari SM, Coyle C, Mortazavi SM, Sharkey PF, Parvizi J. Revision hip arthroplasty: infection is the most common cause of failure. Clin Orthop Relat Res. 2010.

6. Mortazavi SM, Schwartzenberger J, Austin MS, Purtill JJ, Parvizi J. Revision total knee arthroplasty infection: incidence and predictors. Clin Orthop Relat Res. 2010; 468: 2052.

7. Hossain F, Patel S, Haddad F. Midterm assessment of causes and results of revision total knee arthroplasty. Clin Orthop Relat Res. 2010; 468: 1221

8. Pulido L, Ghanem E, Joshi A, Purtill JJ, Parvizi J. Periprosthetic joint infection: the incidence,timing, and predisposing factors. Clin Orthop Relat Res. 2008; 466: 1710.

9. Ha'eri G, Wiley A. Total hip replacement in a laminar flow environment with special reference to deep infections. Clin Orthop Relat Res. 1980; 148: 163.

10. Howorth F. Prevention of airborne infection during surgery. Lancet 1985;325:386.

11. Chan KY, Singh VA, Oun BH, To BH. The rate of glove perforations in orthopaedic procedures: single versus double gloving. A prospective study. Med J Malays. 2006; 61: 3.

12. Ersozlu S, Sahin O, Ozgur AF, Akkaya T, Tuncay C. Glove punctures in major and minor orthopaedic surgery with double gloving. Acta Orthop Belg. 2007; 73: 760.

13. Laine T, Aarnio P. Glove perforation in orthopaedic and trauma surgery. A comparison between single, double indicator gloving and double gloving with two regular gloves. J Bone Joint Surg Br. 2004; 86: 898.

14. Yinusa W, Li YH, Chow W, Ho WY, Leong JC. Glove punctures in orthopaedic surgery. Int Orthop. 2004; $28: 36$. 
15. Thanni LO, Yinusa W. Incidence of glove failure during orthopedic operations and the protective effect of double gloves. J Natl Med Assoc. 2003; 95: 1184.

16. Al-Habdan I, Sadat-Ali M. Glove perforation in pediatric orthopedic practice. J Pediatr Orthop. 2003; $23: 791$.

17. Nicolai P, Aldam CH, Allen PW. Increased awareness of glove perforation in major joint replacement. A prospective, randomised study of Regent Biogel Reveal gloves. J Bone Joint Surg Br. 1997; 79: 371.

18. Maffulli N, Capasso G, Testa V. Glove perforation in elective orthopedic surgery. Acta Orthop Scand. 1989; 60: 565.

19. Partecke LI, Goerdt AM, Langner I, Jaeger B, Assadian O, Heidecke CD, et al. Incidence of microperforation for surgical gloves depends on duration of wear. Infect Control Hosp Epidemiol. 2009; 30: 409.

20. International, A. Standard test method for detection of holes in medical gloves in D5151. ASTM International; 2006.

21. Misteli H, Weber WP, Reck S, Rosenthal R, Zwahlen M, Fueglistaler P et al. Surgical glove perforation and the risk of surgical site infection. Arch Surg. 2009; 144: 553.

22. Myers D, Epling C, Dement J, Hunt D. Risk of sharp device-related blood and body fluid exposure in operating rooms. Infect Control Hosp Epidemiol. 2008; 29: 1139.

23. Tanner J, Parkinson H. Double gloving to reduce surgical cross-infection. Cochrane Database Syst Rev 2006;3:CD003087.

24. Lemaire R, Masson J. Risk of transmission of blood-borne viral infection in orthopaedic and trauma surgery. J Bone Joint Surg Br. 2000; 82: 313 .

25. McCue S, Berg E, Saunders E. Efficacy of double-glovin g as a barrier to microbial contamination during total joint arthroplasty. J Bone Joint Surg. 1981; 63: 811.

26. Laine T, Aarnio P. How often does glove perforation occur in surgery? Comparison between single gloves and a double-gloving system. Am J Surg. 2001; 181: 564. 